\title{
Nanotechnology for multimodality treatment of cancer (Review)
}

\author{
XIAO-YING ZHANG ${ }^{1}$ and PEI-YING ZHANG ${ }^{2}$ \\ ${ }^{1}$ Nanjing University of Chinese Medicine, Information Institute, Nanjing; ${ }^{2}$ Department of Cardiology, \\ Xuzhou Central Hospital, The Affiliated Xuzhou Hospital of Medical College \\ of Southeast University, Xuzhou, Jiangsu 221009, P.R. China
}

Received April 27, 2016; Accepted October 4, 2016

DOI: $10.3892 / \mathrm{ol} .2016 .5322$

\begin{abstract}
Nanotechnology is the latest evolving field and its applications in medicine in recent decades has shown great potential. It has also given a new face to the therapeutics against cancer in recent years. The electronic databases of MEDLINE, EMBASE and PubMed were searched for recent studies, reporting the importance of nanomedicine. The concluding remarks of the above papers mostly confirmed the growing potential of nanomedicine, particularly in the field of cancer. Furthermore, nanomedicine has been observed to promote the therapeutic effect of agents by formulating them into nanocarriers. Delivery of the therapeutic agents via nanodelivery systems is dedicated to solving problems in traditional anticancer agents, including formulation in the physiological environment, their accumulation in tumor, anc their adverse side effect in normal organs. The present review focused on the latest updates on nanotechnology in cancer. In conclusion, the future of any therapeutic option lies in the specific delivery of the particular drug. Additionally, this specific delivery may be achieved efficiently by nanodeliver systems and more studies should be conducted in this direction for the establishment of nanodelivery systems as gold standard delivery modules in clinicalsetting.

\section{Contents}

1. Introduction

2. Approaches of nanomedicine for multimodality treatment of cancer

3. Conclusions

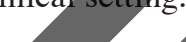

(

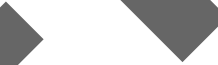

Introduction

Nanomedicine as a multimodality treatment of cancer utilizes the advantages of nanodelivery systems (1). In addition, it is superior to the clinical administration of different therapeutic agents in several aspects, including simultaneous delivery of agents to the active site, precise ratio control of the loading agents and oververcoming multidrug resistance (MDR) (2). Nanodelivery system may simultaneously deliver different agents to the active site, which cannot be achieved by traditional medicine (2). Such simultaneous delivery is able to cause a special interaction between the agents, which cannot be achieved by the administration of different therapeutic agents separately. For example, anti-angiogenesis therapy may decrease vascular leakage and intratumor pressure, which temporarily improves oxygenation, delivery of therapeutic agents and sensitivity to radiation and chemotherapy. However, such favorable change in tumor vasculature is transient (3).

Nanomedicine as a multimodality treatment also allows us to load different therapeutic agents at an exact ratio. More importantly the loading ratio can be sustained until the agents penetrate the membrane of cancer cells and reach their active site with the protection of nanocarriers. For example, Mi et al demonstrated that when combining docetaxel and cisplatin together in nanoparticles, all the formulations with different drug ratios showed an enhanced anticancer effect than the single administration of any of the two drugs at the same total drug concentration (4). Furthermore, nanomedicine for multimodality treatment of cancer is able to overcome MDR, not only through the protection of nanocarriers but also through downregulation of some MDR-related proteins. Currently, siRNA-based nanomedicine is also in use against cancer (5).

\section{Approaches of nanomedicine for multimodality treatment of cancer}

Polymeric nanoparticles. Polymeric nanoparticles are usually prepared with biodegradable polymers and are formed through the entangling of polymers into a dense structure with thermodynamic stability in aqueous solvent. They are used as delivery carriers due to their tunable size, high loading efficiency, easily functionalized surface, high biocompatibility

and bioavailability. Many biodegradable polymers have been
Correspondence to: Dr Pei-Ying Zhang, Department of Cardiology, Xuzhou Central Hospital, The Affiliated Xuzhou Hospital of Medical College of Southeast University, 199 South Jiefang Road, Xuzhou, Jiangsu 221009, P.R. China

E-mail: zpying58@126.com

Key words: nanotechnology, nanomedicine, cancer, nanocarriers 
synthesized in recent years, including the U.S. Food and Drug Administration (FDA)-approved ones, such as polylactic acid (PLA), poly(lactic-co-glycolic acid) 43 (PLGA) and polycaprolactone (PCL). They show several deficiencies including too much hydrophilicity for the encapsulation of hydrophilic drugs and too much mechanical strength for desired release of the encapsulated agents. Polyethylene glycol (PEG)ylated copolymers have been developed to modify their amphiphilicity, reduce the degradation rate, improve performance and increase biocompatibility (6), such as PLA-PEG, PLGA-PEG and PLA-tocopheryl polyethylene glycol succinate (TPGS). Of these, TPGS as a PEGylated vitamin E demonstrated the advantages of both PEG and vitamin E including extending the half-life of the drug in plasma, enhancing the cellular uptake with enhanced anti-cancer effects $(7,8)$. As multimodality treatment vehicles, polymeric nanoparticles could load agents in their core or be coated with the agents on the surface by chemical conjugation or physical adsorption. Compared with other delivery systems, such as micelle or liposomes, polymeric nanoparticles show higher stability in aqueous solvent and more desirable controlled release due to their dense structure.

Polymeric micelles. Polymeric micelles show technical ease, high biocompatibility and high efficiency in drug delivery. They also show advantages in passively targeting the tumor area through enhanced permeability and retention (EPR) effect, due to their small size ranging from 10 to $100 \mathrm{~nm}$. The application of micelles in drug delivery often achieves favorable biodistribution, higher therapeutic effects and lower side effects for the delivered therapeutic agents. Multimodality treatment may be achieved with micelles by loading hydro phobic therapeutic agents in their cores or by conjugating/ electrostatically adsorbing the hydrophilic therapeutic agents to their shells.

Cho et al delivered multi-drugs for combinatory chemotherapy with poly(ethylene glycol)-b-poly ( $\varepsilon$-caprolactone) micelles (9). Another three-in-one micelles loading, i.e., paclitaxel, 17-allylamino-17-demethoxygeldanamycin (17-AAG) and rapamycin were developed by Shin et al with poly(ethylene glycol)-b-poly(D,L-lactic acid) (PEG-b-PLA). The micelles exerted strong synergy in MCF-7 and 4T1 breast cancer cells by analyzing their combination index (10).

Liposomes. Liposomes are vesicles composed of lipid bilayers with water in their interior. They are a mimic of the cell membrane and one of the earliest FDA-approved drug delivery systems due to their high biocompatibility. Compared with polymeric micelles or nanoparticles, liposomes showed advantages in loading both hydrophilic and hydrophobic agents, especially with high loading efficiency for hydrophilic ones. However, liposomes suffer many problems such as large size, low stability of structure, low loading efficiency and undesired release profile compared with other delivery systems. To improve the performance of liposomes, polymeric liposomes are developed with better stability, desirable controlled release, as well as functional group for further decoration. Treatment with liposomes containing the two drugs led to the appearance of necrosis in almost all the tumors (91\% response), indicating significant improvement in efficacy (11).
Nanohydrogels. Nanohydrogels are 3D networks of cross-linked hydrophilic polymers with high water content and with a size usually of $<200 \mathrm{~nm}$. They combine the advantages of hydrogels and nanoparticles for drug formulation and delivery. The cross-linking of nanohydrogels is either through physical interaction such as hydrophilic-hydrophobic interactions, electrostatic interactions or hydrogen bonding, or through chemical interaction with covalent bond (12). The swelling of nanohydrogels in the aqueous environment is determined by type of polymers, degree of cross-linking and external environment (13). Nanohydrogels are promising in the delivery of therapeutic agents for multimodality treatment, especially for hydrophilic biomolecules such as peptides, proteins and oligonucleotides. They show high biocompatibility due to their high water content (14). The positively charged surface could then load negatively charged proteins for co-delivery. The nanogels showed efficient cell uptake (15). The gels used to co-deliver paclitaxel and doxorubicin showed significant prolongation of the surviyal rate in B16-F10 tumor-bearing mice (16).

Dendrimers. Dendrimers are symmetrical and spherical macromolecules, exhibiting a tree-like structure with numerous dendritic arms stretching from a core. The number of branching shells is denoted as the generation $(G)$. Compared with other delivery systems, dendrimers often show advantages in size uniformity, rapid cellular entry, reduced macrophage uptake, target ability and more facile passage across biological barriers by transcytosis $(17,18)$. Their reactive surface with abundant functional groups makes them easier to load different therapeutic agents efficiently through conjugation. In addition, their interior shows solvent-filled orid space for drug encapsulation. Therefore, dendrimers are promising nanodelivery system for multimodality treatment. T7-dendrimers showed a decrease of tumor volume by $77.09 \%$ compared to the control group, while DOX-loaded T7-dendrimers, DNA-loaded T7-dendrimers inhibited tumor growth by 41.49 and $46.89 \%$ at the same dose, respectively, demonstrating the advantages of the combination of chemotherapy and gene therapy (19).

Solid lipid nanoparticles (SLNS). SLNs are a class of lipospherical nanocarriers with an average diameter of $10-1,000 \mathrm{~nm}$, formed with lipids that remain in a solid state at room temperature and body temperature (20). They combine the advantages of liposomes and polymeric nanoparticles, and show high stability in the physiological environment. Their preparation processes usually do not need to induce toxic organic solvents. They can load both hydrophilic and hydrophobic agents, especially showing advantages in protein or peptide delivery. They also show good potential for large-scale production (21). The CI value of PQSLN/Bcl-2 siRNA was smaller than 0.5. It also showed that the platform synergistically promoted the apoptotic death of cancer cells by activating caspase-mediated pathways (22). Cell viability studies in MIA PaCa-2 and Panc-1 cells demonstrated that the combination of low doses of the drugs significantly reduced cell viability by 43.6 and $48.49 \%$, respectively, which showed the superiority of the multimodality treatment to the individual treatment (23). 
Inorganic nanoparticles. Apart from the above organic delivery systems, mainly composed of polymers or lipids, there are other types of delivery systems composed of inorganic materials, including carbon nanotubes or graphene oxide, gold nanoparticles, and magnetic nanoparticles such as iron oxide nanoparticles. They are studied as nanodelivery systems for the multimodality treatment of cancer due to their unique image or thermal properties. Silica nanoparticles are also utilized as nanocarriers due to their high stability, high biocompability, high uniformity as well as fabrication and modification ease. These nano-sized particles are able to load other therapeutic agents via physical interaction or co-valence bond for multimodality treatment. Carbon nanotubes or graphene oxide are able to deliver therapeutic agents as well as to generate fatal heat upon NIR irradiation (24). Magnetic nanoparticles, such as superparamagnetic iron oxides, are another type of commonly used agent for thermotherapy. They can generate localized heat to destroy cancer cells under exposure to an alternating magnetic field (25).

Hybrid nanocarriers. Hybrid nanocarriers are a combination of formerly mentioned nanodelivery systems. Each of the systems has both advantages and disadvantages regarding agent load, agent encapsulation efficiency, cellular uptake, pharmacokinetics and biodistribution. Hybrid nanocarriers achieve better delivery efficiency as well as therapeutic effect for multimodality treatment. Additionally, the hybrid carriers provide a broad possibility to load multi-types of therap agents together (26).

Another type of hybrid nanocarrier nanoparticles or micelles. Prodrug nanopar PLA-cisplatin, PLA-doxorubicin or PLA also produced for synergistic multidrug polyplexes showed higher anticancer potency than paclitaxel alone (28).

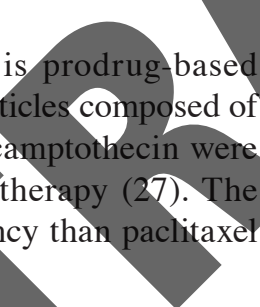

TPGS. Structure of TPGS: D- $\alpha$-tocopheryl polyethylene glycol 1,000 succinate (vitamin E TPGS) is a water-soluble derivative of natural vitamin $\mathrm{E}$, which is formed by esterification of vitamin E succinate with PEG. It has the advantages of both PEG and vitamin $E$ in the application of various drug delivery devices, including extending the half-life of the drug in plasma and enhancing its cellular presence. TPGS has an amphiphilic structure. TPGS has also been served as the excipient for overcoming MDR and as the inhibitor of P-glycoprotein (P-gp) for increasing the oral bioavailability of anticancer drugs. TPGS-based nanocarriers can significantly enhance the solubility, permeability and stability of the formulated drugs. TPGS has been proven to be an efficient emulsifier for the synthesis of nanoparticles of biodegradable polymers leading to high cellular uptake and high therapeutic effects (7).

\section{Conclusions}

It can be concluded from the above observations that studies concerning nanotechnology as an efficient delivery system against cancer are being conducted. Thus, the future of drug delivery lies mainly in nanodelivery systems.

\section{References}

1. Omlor AJ, Nguyen J, Bals R and Dinh QT: Nanotechnology in respiratory medicine. Respir Res 16: 64, 2015.

2. Taymouri S and Varshosaz J: Effect of different types of surfactants on the physical properties and stability of carvedilol nano-niosomes. Adv Biomed Res 5: 48, 2016.

3. Ferrara $\mathrm{N}$ and Kerbel RS: Angiogenesis as a therapeutic target. Nature 438: 967-974, 2005.

4. Mi Y, Zhao J and Feng SS: Targeted co-delivery of docetaxel, cisplatin and herceptin by vitamin E TPGS-cisplatin prodrug nanoparticles for multimodality treatment of cancer. J Control Release 169: 185-192, 2013.

5. Zhao J, Mi Y and Feng SS: siRNA-based nanomedicine. Nanomedicine (Lond) 8: 859-862, 2013.

6. Zhang Z, Yang X and Feng SS: Copolymer technology for advanced nanomedicine. Nanomedicine (Lond) 6: 583-587, 2011.

7. Zhang Z, Tan S and Feng SS: Vitamin E TPGS as a molecular biomaterial for drug delivery. Biomaterials 33: 4889-4906, 2012.

8. Mi Y, Liu Y and Feng SS: Formulation of Docetaxel by folic acid-conjugated d- $\alpha$-tocopheryl polyethylene glycol succinate 2000 (Vitamin E TPGS(2k)) micelles for targeted and synergistic chemotherapy, Biomaterials 32: 4058-4066, 2011.

9. Cho H, Lai TC and Kwon GS: Poly(ethylene glycol)-block-poly( $\varepsilon-$ caprolactone) micelles for combination drug delivery: evaluation of pachitaxel, cyclopamine and gossypol in intraperitoneal xenograft models of ovarian cancer. J Control Release 166: $1-9$.

10. Shin HC, Alani AWG, Cho H, Bae Y, Kolesar JM and Kwon GS: A -in-1 polymeric micelle nanocontainer for poorly water-soluble drugs. Mol Pharm 8: 1257-1265, 2011.

Zucker D, Andriyanoy AV, Steiner A, Raviv U and Barenholz Y: haracterization of PEGylated nanoliposomes co-remotely loaded with topotecan and vincristine: relating structure and pharmacokinetics to therapeutic efficacy. J Control Release 160: 281-289, 2012.

Yallapu MM, Jaggi M and Chauhan SC: Design and engineering of namogels for cancer treatment. Drug Discov Today 16: 457-463, 2011.

Kabanov AV and Vinogradov SV: Nanogels as pharmaceutical carriers: finite networks of infinite capabilities. Angew Chem Int Ed Engl 48: 5418-5429, 2009.

14. Qian ZY, Fu SZ and Feng SS: Nanohydrogels as a prospective member of the nanomedicine family. Nanomedicine (Lond) 8: 161-164, 2013.

15. González-Toro DC, Ryu JH, Chacko RT, Zhuang J and Thayumanavan S: Concurrent binding and delivery of proteins and lipophilic small molecules using polymeric nanogels. J Am Chem Soc 134: 6964-6967, 2012.

16. Zhao L, Zhu L, Liu F, Liu C, Shan-Dan, Wang Q, Zhang C, Li J, Liu J, Qu X, et al: pH triggered injectable amphiphilic hydrogel containing doxorubicin and paclitaxel. Int J Pharm 410: 83-91, 2011.

17. Menjoge AR, Kannan RM and Tomalia DA: Dendrimer-based drug and imaging conjugates: design considerations for nanomedical applications. Drug Discov Today 15: 171-185, 2010.

18. Lee CC, MacKay JA, Fréchet JMJ and Szoka FC: Designing dendrimers for biological applications. Nat Biotechnol 23: 1517-1526, 2005.

19. Han L, Huang R, Li J, Liu S, Huang S and Jiang C: Plasmid pORF-hTRAIL and doxorubicin co-delivery targeting to tumor using peptide-conjugated polyamidoamine dendrimer. Biomaterials 32: 1242-1252, 2011.

20. Muller RH, Mader K and Gohla S: Solid lipid nanoparticles (SLN) for controlled drug delivery - a review of the state of the art. Eur J Pharm Biopharm 50: 161-177, 2000.

21. Almeida AJ and Souto E: Solid lipid nanoparticles as a drug delivery system for peptides and proteins. Adv Drug Deliv Rev 59: 478-490, 2007.

22. Bae KH, Lee JY, Lee SH, Park TG and Nam YS: Optically traceable solid lipid nanoparticles loaded with siRNA and paclitaxel for synergistic chemotherapy with in situ imaging. Adv Healthc Mater 2: 576-584, 2013.

23. Sutaria D, Grandhi BK, Thakkar A, Wang J and Prabhu S: Chemoprevention of pancreatic cancer using solid-lipid nanoparticulate delivery of a novel aspirin, curcumin and sulforaphane drug combination regimen. Int J Oncol 41: 2260-2268, 2012. 
24. Mahmood M, Karmakar A,Fejleh A, Mocan T, Iancu C, Mocan L, Iancu DT, Xu Y, Dervishi E, Li Z, et al: Synergistic enhancement of cancer therapy using a combination of carbon nanotubes and anti-tumor drug. Nanomedicine (Lond) 4: 883-893, 2009.

25. Barick KC, Singh S, Jadhav NV, Bahadur D, Pandey BN and Hassan PA: pH-responsive peptide mimic shell cross-linked magnetic nanocarriers for combination therapy. Adv Funct Mater 22: 4975-4984, 2012

26. Zheng M, Yue C, Ma Y, Gong P,Zhao P,Zheng C, Sheng Z,Zhang P, Wang $\mathrm{Z}$ and Cai L: Single-step assembly of DOX/ICG loaded lipid - polymer nanoparticles for highly effective chemo-photothermal combination therapy. ACS Nano 7: 2056-2067, 2013.
27. Aryal S, Hu CM and Zhang L: Polymeric nanoparticles with precise ratiometric control over drug loading for combination therapy. Mol Pharm 8: 1401-1407, 2011.

28. Nam K, Nam HY, Kim PH and Kim SW: Paclitaxel-conjugated PEG and arginine-grafted bioreducible poly (disulfide amine) micelles for co-delivery of drug and gene. Biomaterials 33: 8122-8130, 2012.

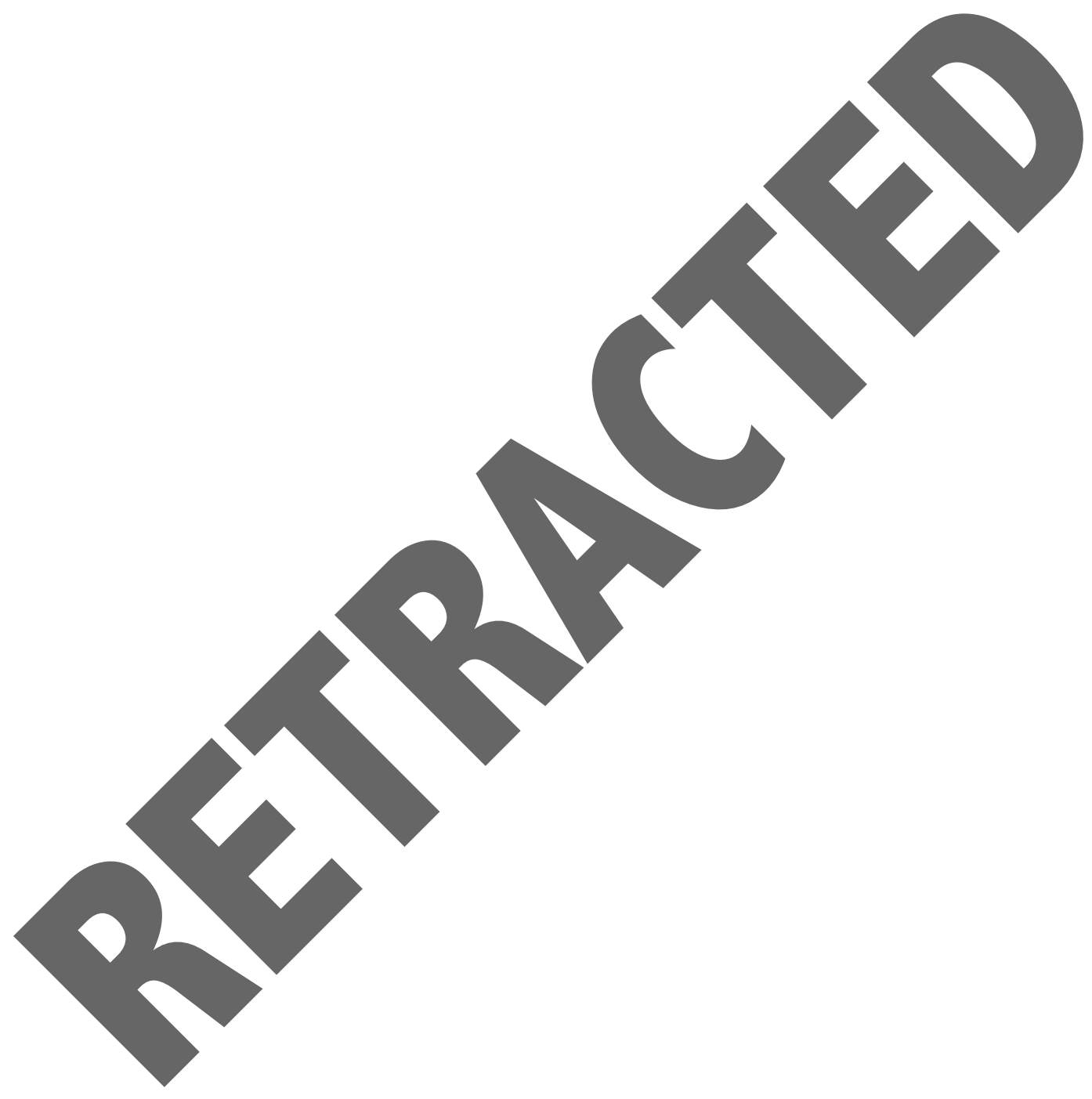

